\title{
Plotin, la mémoire et la connaissance des intelligibles
}

Riccardo Chiaradonna

\section{(2) OpenEdition}

1 Journals

Édition électronique

URL : https://journals.openedition.org/philosant/2473

DOI : $10.4000 /$ philosant.2473

ISSN : 2648-2789

Éditeur

Éditions Vrin

\section{Édition imprimée}

Date de publication : 13 novembre 2009

Pagination : 5-33

ISBN : 978-2-7574-0124-8

ISSN : 1634-4561

\section{Référence électronique}

Riccardo Chiaradonna, " Plotin, la mémoire et la connaissance des intelligibles », Philosophie antique [En ligne], 9 | 2009, mis en ligne le 25 juillet 2019, consulté le 02 décembre 2022. URL : http:// journals.openedition.org/philosant/2473; DOI : https://doi.org/10.4000/philosant.2473

\section{(c) $(1) \&$}

Creative Commons - Attribution - Pas d'Utilisation Commerciale - Pas de Modification 4.0 International - CC BY-NC-ND 4.0

https://creativecommons.org/licenses/by-nc-nd/4.0/ 


\section{PLOTIN, LA MÉMOIRE ET LA CONNAISSANCE DES INTELLIGIBLES*}

Riccardo CHIARADONNA

Université de Rome 3

RÉSUMÉ. Plotin discute à plusieurs reprises de la mémoire, de sa fonction et de son rôle dans la connaissance. Dans la longue section qui va de IV 3 [27], 25 à IV 4 [28], 5, il s'interroge sur «ce qui » se remémore, c'est-à-dire sur le sujet de la mémoire. L'examen de ces chapitres permet de recueillir quelques éléments caractéristiques de la théorie plotinienne de la connaissance. Plotin fait une rigoureuse distinction entre la mémoire et la simple conservation d'impressions sensibles et associe la mémoire à une capacité spontanée de l'âme. L'âme, d'autre part, en tant que sujet de la mémoire, est pourtant toujours caractérisée par un type de pensée discursif et lié au temps. De ce type de connaissance, Plotin distingue la pensée la plus élevée, qui appartient à l'âme grâce à la partie d'elle-même qui n'est pas descendue de l'Intellect, lequel est étranger au temps et à la discursivité. L'étude du sujet de la mémoire fournit des éclaircissements sur la structure de l'âme chez Plotin, sur le rôle de l'imagination (phantasia), sur le caractère intrinsèquement dynamique de l'âme, capable de redéfinir sa propre nature en accord avec la faculté qui joue en elle le rôle de centre unificateur. Au sommet se trouve une condition cognitive dans laquelle l'âme saisit, même «icibas », avant sa séparation du corps, les Formes intelligibles, au moyen d'une connaissance appropriée et indépendante de toute forme de mémoire et de discursivité ( cf. IV 4 [28], 4-5). Par conséquent, le rôle de la réminiscence dans l'épistémologie plotinienne est plutôt limité.

SUMMARY. On several occasions Plotinus discusses memory : its function and relation to knowledge. In the long section that runs from IV 3 [27], 25 to IV 4 [28], 5, Plotinus considers what it is that remembers, i.e. what constitutes the subject of memory. An examination of these chapters reveals a number of features in the Plotinian theory of knowledge. Plotinus draws a thorough distinction between memory and the mere conservation of sense data, and links the former to an intrinsic power of the soul. The Soul, however, as the

\footnotetext{
${ }^{*}$ Cette étude est une version revue et corrigée sur de nombreux points d'un article à paraître dans Pieretti (à paraitre). Je tiens à remercier vivement Silvia Ferretti et Daniela Taormina pour avoir lu cet article avant publication. Michel Narcy a bien voulu le traduire en français et en a amélioré beaucoup de détails ; qu’il en soit lui aussi remercié ici.
}

Philosophie antique, $\mathrm{n}^{\circ} 9$ (2009), 5-33 
subject of memory, is always characterized by some kind of discursive thought, and bound up with time. Plotinus distinguishes this kind of knowledge from the highest thought, which belongs to the part of the Soul that has not descended from the Intellect, and is extraneous to time and discursivity. In addressing the issue of the subject of memory, Plotinus also draws light on the structure of the Soul and the role of imagination (phantasia). Soul emerges as an intrinsically dynamic essence, which has the power of redefining its own nature in concert with the faculty which acts as its unifying centre. At the bighest level is a cognitive condition in which the Soul, even "bere below", prior to its separation from the body, gathers the intelligible Forms by means of an appropriate knowledge, independent of any kind of memory and discursivity (see IV 4 [28], 4-5). As a result, the role of recollection in Plotinian epistemology is rather limited. 
Ennéades exigerait probablement tout un livre ; je me bornerai ici à mettre en lumière certains points que les recherches précédentes n'ont pas toujours dégagés.

Dans le traité Sur les difficultés relatives à l'âme, Plotin soulève la question de savoir si les âmes qui ont abandonné le corps conservent ou non, après qu'elles l'ont quitté, la faculté de se souvenir. Pour mener correctement l'enquête sur ce point, note-t-il (IV 3 [27], 25.6-8), il est nécessaire

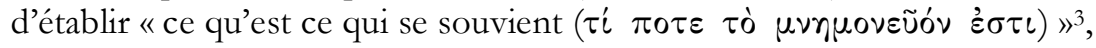

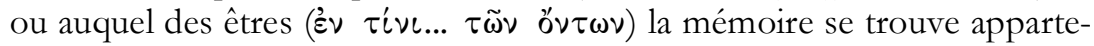

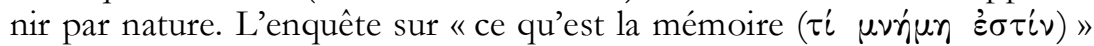
(25.7) est en revanche explicitement écartée parce que, observe Plotin, on en a traité ailleurs et souvent discuté (25.8-10).

Ces lignes soulèvent un certain nombre de problèmes. En premier lieu, il convient de s'arrêter sur les deux questions distinguées par Plotin : (i) qu'est-ce que la mémoire ? (ii) quel est le sujet de la mémoire, qu'estce qui, par nature, est fait pour exercer la mémoire ? On suppose généralement que la référence à ce qui a été développé «ailleurs » (IV 3 [27],

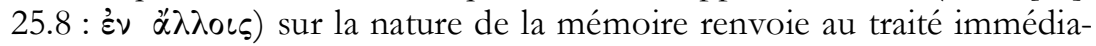
tement précédent dans l'ordre chronologique, à savoir III 6 [26] Sur l'impassibilité des incorporels. On trouve là, en effet, une allusion à la mémoire, dont Plotin souligne la nature active, qui renvoie à une capacité de l'âme (autrement dit, d'une substance incorporelle) ${ }^{4}$ et qui ne peut être réduite à la simple conservation des impressions sensibles :

... on l'a dit, les souvenirs non plus ne résultent pas de ce que quelque chose vient à se déposer dans l'âme, mais de ce qu'elle-même éveille sa capacité ( $\delta u ́ v a u \iota v)$, de sorte que, dans ce cas aussi, elle possède aussi ce qu'elle ne possède pas. (III 6 [26], 2.42-44.)

3. Les traductions des passages des Ennéades sont les miennes.

4. Il faut rappeler que pour Plotin l'âme admet différentes divisions internes: en général, les spécialistes pensent qu'il distingue (i) l'âme comme principe métaphysique universel; (ii) l'âme du monde; (iii) l'âme individuelle ou l'âme de chaque homme. $C f$. Blumenthal 1971 (b). Comme on le verra à propos de l'âme individuelle, chacune de ces divisions est articulée à son tour selon plusieurs niveaux. Il faut enfin ajouter à cette classification les âmes des astres, dont la condition cognitive supérieure diffère sensiblement de celle des âmes individuelles descendues dans les corps ( $c f$. IV 4 [28], 6 sqq.). Il faut rappeler, par ailleurs, que les divisions de l'âme selon Plotin ne doivent pas s'entendre comme des partitions quasi-sensibles ou en tout cas quantitatives (IV 3 [27], 2). Dès ses premiers traités ( $c$. IV 9 [8]), Plotin affronte le problème d'établir si toutes les âmes n'en sont qu'une. En fait, l'âme de l'univers et les âmes individuelles sont toutes des «parties » congénères d'une âme totale unique, et chaque âme est à la fois elle-même et "toute » lâme. La distinction des niveaux ne doit donc pas être conçue comme une classification rigide; il n'est pas rare que Plotin, dans ses explications, passe de façon plutôt désinvolte de l'un à l'autre «type » d'âme. Cf. Chiaradonna 2009, p. 52-55. 
Les interprètes ont vu en Aristote (Mem. 1, 450a25 sqq.) et en les stoïciens (SVF II 847) les adversaires contre qui polémique Plotin en défendant cette conception anti-empiriste de la mémoire ${ }^{5}$. C'est principalement (mais, selon toute probabilité, pas exclusivement) contre le traité aristotélicien De memoria qu'est dirigée la discussion du traité plus tardif IV 6 [41] Sur la sensation et la mémoire 6 . Il faut rappeler que pour Plotin la perception elle-même ne consiste pas en une impression reçue par l'âme, mais implique de la part de cette dernière une capacité active : par conséquent, la perception est assimilée, non à une altération (l'âme, en tant que substance incorporelle, n'est pas sujette à altération), mais à un juge-

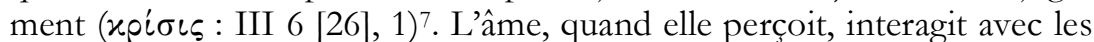
objets sensibles, sans que cela implique pour autant qu'elle soit affectée par eux ; si quelque chose est affecté, c'est le corps animé (ce que Plotin, dans le traité tardif I 1 [53], appelle « le vivant»), non pas l'âme qui lui est préposée et qui est une substance existant par elle-même. Si donc la perception est en elle-même active et implique une capacité d'ordre intelligible, à plus forte raison devra-t-on en dire autant de la mémoire :

Si nous disons que les sensations ne sont pas des impressions ( $\tau u \pi \omega \dot{\sigma \varepsilon \iota \varsigma) ~}$ et ne s'impriment pas dans l'âme comme les empreintes d'un sceau

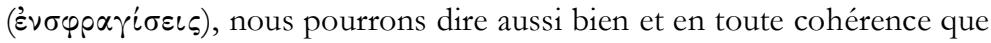
les souvenirs non plus ne coïncident pas avec la conservation de nos connaissances et de nos sensations grâce à la persistance de l'empreinte dans l'âme : cette empreinte, en effet, n'y existait pas dès le début. (IV 6 [41], 1.1-5.)

Cependant, l'allusion à la nature de la mémoire de III 6 [26], 2.42 sqq. paraît d'autant moins propre à justifier le renvoi de IV 3 [27], 25.8-9 qu'en III 6 [26], 2 aussi Plotin semble renvoyer à un développement

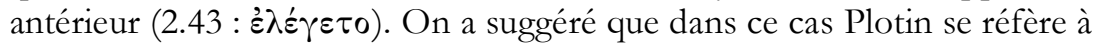
une brève section du traité IV 7 [2] Sur l'immortalité de l'âme ${ }^{8}$, mais peutêtre n'est-il pas à exclure, comme l'a observé Barrie Fleet, que Plotin renvoie - en III 6 [26], 2 comme en IV 3 [27], 25 -, non pas à un traité des Ennéades, mais aux discussions de son « séminaire »" ce que semblent en effet suggérer les mots «on en a souvent discuté ( $\pi 0 \lambda \lambda \dot{\alpha} x \iota \varsigma$ $\tau \varepsilon \vartheta \rho u ́ \lambda \lambda \eta \eta \tau \iota)$ » de IV 3 [27], 25.9.

5. Cf. Fleet 1995, p. 98-99.

6. Sur le rapport complexe entre les conceptions plotinienne et aristotélicienne de la mémoire, voir Morel 2007, p. 378-380.

7. La littérature secondaire sur cette question est abondante; l'étude de référence est Emilsson 1988.

8. Cf. IV 7 [2], 6.43-49: cf. Henry \& Schwyzer 1964 ad III 6 [26], 2.42.

9. Cf. Fleet 1995, p. 98. 
Les premières lignes de la section sur la mémoire en IV 3 [27] doivent nous retenir encore parce que, comme il arrive plusieurs fois dans les Ennéades, Plotin, au début de la discussion, en donne sommairement une première exposition générale où l'on trouve les éléments principaux du traitement de la question qui va suivre ${ }^{10}$. Il est d'une importance cruciale d'observer que Plotin, au début de sa recherche sur le sujet de la mémoire, est avant tout soucieux d'établir que le Dieu intelligible, autrement dit l'Intellect hypostase dont le contenu de pensée est identique avec les Formes intelligibles, ne se souvient pas. Cette thèse oriente toute la discussion qui suit, et il est indispensable de l'avoir constamment à l'esprit :

Si ce qui appartient à la mémoire est quelque chose d'acquis (๕̇ $\pi \iota x \tau \dot{\imath} \tau o u$ $\tau \iota \nu o ́ \varsigma)$, qu'il s'agisse d'une connaissance apprise ( $\mu \alpha \vartheta \dot{n} \mu \alpha \tau \circ \varsigma)$ ou d'une affection $(\pi \alpha \vartheta \dot{\eta} \mu \alpha \tau \circ \varsigma)$, le fait de se souvenir ne se rencontrera ni dans les êtres impassibles ni en ceux qui ne sont pas dans le temps. Nous ne devons assurément situer la mémoire ni en Dieu ni en ce qui est Être et Intellect: rien, en effet, ne leur vient de l'extérieur, et ce n'est pas le temps, mais l'éternité qui environne l'Être : en lui ne se trouve ni l'avant ni l'après, mais il est toujours tel qu'il est, dans le même état, sans accueillir de changement. (IV 3 [27], 25.10-17.)

On retrouve ici des thèses bien connues de la métaphysique plotinienne. Platon, dans le Timée (37e-38a), affirme qu'à l'essence " éternelle »

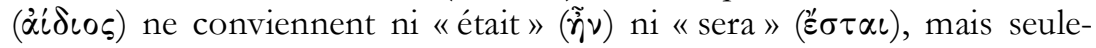

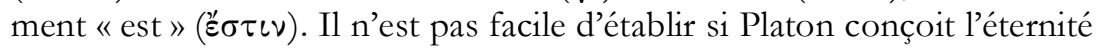
de manière non durative : la question est débattue et les spécialistes n'ont pas atteint de conclusions qui fassent l'unanimité. Quoi qu'il en soit, plusieurs philosophes platoniciens de l'antiquité ont soutenu, en s'appuyant sur le Timée, que l'être intelligible est situé dans un présent supérieur à la durée temporelle. Cette conception est exprimée dans le De E apud Delphos de Plutarque (19, 392E-20, 393A), dans le discours d'Ammonios qui nie que Dieu soit «accordé au temps $»^{11}$. Dans le traité plotinien III 7 [45] L'éternité et le temps, la doctrine de l'éternité comme présent atemporel trouve son expression achevée ${ }^{12}$ : le temps naît seulement au niveau ontologique de l'âme, dont l'activité n'a pas lieu «toute ensemble » mais implique une succession ordonnée de moments (cf. III 7 [45], 11.35-40) ${ }^{13}$. Une conception semblable est le présupposé le plus évident de l'argumentation de Plotin en IV 3 [27], 25 : l'Intellect divin, la deuxième

10. Sur cette structure argumentative, $c f$. Chiaradonna 2002, p. 156.

11. Voir la discussion dans Ferrari 1995, p. 57-58 et, sur la notion d'atemporalité, les précisions de Burnyeat 2005, p. 166.

12. Cf. Beierwaltes 1981, p. 35-49.

13. Plus de détails dans Chiaradonna 2008 (a). 
hypostase, n'est pas dans le temps; par conséquent, il ne pourra posséder la mémoire, puisque son type de pensée n'admet pas l'avant et l'après ; son activité se déploie dans un présent éternel sans durée. Significativement, Plotin ne fait ici aucune allusion à l'Un, le premier principe d'où tire son origine l'Intellect hypostase lui-même. Cela n'est pas surprenant : l'Un, en effet, est absolument simple et privé de toute forme de pensée, même de la pensée intuitive et atemporelle de l'Intellect. Dans la discussion sur le sujet de la mémoire, en revanche, il est important d'établir quel type de pensée est associé au souvenir, lequel n'y est pas associé, et à quelles réalités doivent être rapportées ces différentes activités cognitives.

L'absence de mémoire qui caractérise l'Intellect est associée non seulement au fait qu'il est hors du temps, mais aussi à un autre caractère particulier de sa nature: l'impassibilité ( $\dot{\alpha} \pi \dot{\alpha} \vartheta \varepsilon \sigma \iota$, IV 3 [27], 25.12). La mémoire est liée à un aspect "passif », au sens large, dont le Nous est exempt. Pareille assertion suscite cependant certaines interrogations. Comme on l'a dit, en effet, la mémoire ne peut être en aucune manière réduite à la conservation d'impressions corporelles reçues : en IV 6 [41], Plotin en met en relief le caractère intrinsèquement actif. Mais s'il en est ainsi, on ne voit pas pourquoi le fait d'être impassible devrait impliquer de ne pas posséder la mémoire : si la mémoire n'est pas une affection passive, alors le sujet qui se remémore peut très bien être exempt d'affections. En outre, en III 6 [26], 1.28-30, il est souligné que l'âme est une substance incorporelle et qu'elle ne peut donc être en aucune façon sujette à des altérations et à des mouvements qui compromettraient son incorruptibilité et son caractère intelligible.

À première vue, tout cela complique passablement l'argumentation de IV 3 [27], 25.10 sqq. : en effet, alors que l'atemporalité sert clairement à distinguer le Dieu intelligible du sujet de la mémoire, la situation est différente en ce qui concerne l'impassibilité. Plotin, comme on vient de le voir, attribue l'impassibilité non seulement à l'Intellect mais aussi à l'âme, qui ressort de la discussion de IV 3 [27] comme le sujet véritable de la mémoire. Deux solutions peuvent être proposées. La première tendrait à reconnaître dans la discussion du traité 27 une façon de concevoir l'âme et la mémoire différente de celle des autres traités et, en particulier, de IV 6 [41]. Bien qu'il souligne généralement le caractère actif de la mémoire et le fait que l'âme est exempte d'affections, en IV 3 [27] Plotin soutiendrait que l'âme est, en dernière analyse, passive et que le sujet qui se remémore (l'âme) est par conséquent susceptible d'être affecté. Une telle conclusion pourrait sembler confirmée aussi par un autre indice : alors qu'en IV 3 [27], 29.24 Plotin semble associer la mémoire à la «con- 


\section{Le sujet de la mémoire}

La discussion sur la mémoire tient une place importante dans les Ennéades de Plotin. Sont en effet consacrés à la mémoire une longue section à la jonction des traités IV 3 [27] et IV 4 [28], Sur les difficultés relatives à l'âme I et II (IV 3 [27], 25-32 ; IV 4 [28], 1-5 - mais la discussion continue jusqu'à IV 4 [28], 17) ${ }^{1}$ - ainsi que, en totalité, le traité IV 6 [41] Sur la sensation et la mémoire, sans compter des indications plus brèves dans d'autres écrits. Il s'agit pourtant d'un thème qui n'a encore été que relativement peu exploré par les spécialistes; peu nombreux sont les travaux qui y sont consacrés, et la relation de la doctrine de la mémoire avec les thèmes principaux de la philosophie plotinienne reste, au moins en partie, à déterminer ${ }^{2}$. Dans cette contribution, je m'appuierai principalement sur les traités IV 3 [27] et IV 4 [28], pour essayer d'éclaircir la place de la mémoire dans la doctrine plotinienne de la connaissance et, en particulier, le rôle que joue la mémoire dans la connaissance des Formes intelligibles. Une analyse adéquate de cette longue et difficile section des

1. Ces deux traités appartiennent, en réalité, à un long écrit unique, comprenant les traités IV 3 [27], IV 4 [28], IV 5 [29], qui fut divisé en trois par Porphyre dans son édition des Ennéades; la coupure entre IV 3 [27] et IV 4 [28], qui intervient à IV 3 [27], 32.27, est particulièrement brutale et arbitraire, car elle interrompt une phrase qui se poursuit en IV 4 [28], 1.1. Dans la présente étude je ne m'arrêterai pas sur les chapitres IV 4 [28], 6 sqq., où est débattue la question de savoir si les astres et l'âme du monde possèdent la mémoire (voir à ce sujet Majumdar 2007, p. 57-58). Je crois en effet que la base doctrinale sur laquelle est fondée l'argumentation de ces chapitres est déjà entièrement explicitée dans la section précédente, où Plotin sépare la mémoire et la discursivité de la connaissance directe des intelligibles propre à l'âme supérieure.

2. Parmi les principales études consacrées à ce sujet, je signale: Warren 1965 ; Blumenthal 1971 (a), p. 80-99 ; Guidelli 1988 ; Brisson 2006 ; D’Ancona 2007 ; Taormina (à paraitre). On peut trouver des approches plus synthétiques dans Gerson 1994, p. 180183 ; Peroli 2003, p. 267-272 ; Remes 2007, p. 111-119; ainsi que dans divers exposés d'ensemble de la philosophie de Plotin. À consulter également, quelques récentes traductions commentées : Brisson 2005 ; Morel 2007. 
servation" ( $\left.\varkappa \propto \tau \circ \chi \chi^{\prime}\right)$ de l'objet de la sensation ${ }^{14}$, en IV 6 [41], 1.3 il nie catégoriquement que les souvenirs consistent en la conservation des connaissances et des sensations. Je crois cependant que de telles contradictions sont seulement apparentes et indiquent, non pas du tout un changement dans la conception de la mémoire, mais seulement une différence d'accent dans la façon dont sont menées les discussions. En IV 3 [27], exactement comme dans les autres traités, Plotin établit une rigoureuse distinction entre les processus qui ont lieu dans l'âme et des processus d'ordre physique qui impliqueraient une altération. En IV 3 [27], 26.25 sqq. il examine de façon critique l'hypothèse qui voudrait que l'âme, du fait qu'elle est dans un corps, en soit en quelque sorte contaminée et rendue comme lui qualifiée - " physicisée ", pour ainsi dire - au point de pouvoir recevoir les empreintes des objets sensibles. Plotin conteste une telle hypothèse au moyen d'une argumentation passablement longue et complexe, dont le premier pas consiste à faire une distinction entre les «empreintes » qui peuvent parvenir à l'âme et des empreintes physiques et corporelles. Tout processus psychique, en effet, est assimilable à un

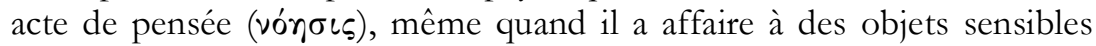

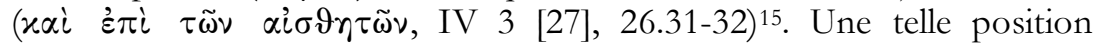
correspond entièrement à celle qui est exposée au début de IV 6 [41], où Plotin nie que la mémoire consiste en la conservation de connaissances et de sensations grâce à la persistance de leurs empreintes dans l'âme (IV 6 [41], 1.4-5).

Si différence il y a entre les deux traités, elle ne porte pas sur les thèses qui y sont soutenues mais, comme on l'a déjà dit, sur la perspective dans laquelle ils se placent: dans IV 3 [27], 25 sqq. Plotin se propose en premier lieu, non pas d'expliquer «ce qu'est la mémoire», mais «ce qu'est ce qui se remémore ». Pour cette raison, à la différence de ce qui se passe en IV 6 [41], une grande importance y est accordée à la distinction entre le mode de pensée de l'Intellect transcendant (et, comme nous le verrons, de l'âme supérieure, non descendue de l'Intellect), qui ne se remémore pas, et le mode de pensée de l'âme sujette au temps et à la mémoire. Par comparaison avec la pensée intuitive de l'Intellect, l'âme qui se remémore peut sans aucun doute être considérée comme « réceptive ", sans pourtant que cela implique qu'elle soit sujette à des altérations en recevant des impressions corporelles. Comme il le fait ailleurs, Plotin, quand il veut distinguer un niveau de réalité du niveau supérieur, finit par associer au niveau inférieur des caractères qui, en rigueur de termes, ne devraient pas lui appartenir, mais qui servent en tout cas à le différencier

14. L'interprétation de ce passage est particulièrement complexe et controversée. Voir Taormina (à paraittre).

15. Voir à ce sujet Emilsson 1988, p. 74-82. 
du principe le plus élevé. L'exemple le plus connu de cette façon d'argumenter concerne l'attribution à l'Intellect de caractères qui impliquent discursivité et imperfection quand il s'agit de le distinguer de l'Un (cf. V 5 [32], 12 ; V 3 [49], 10). Si, en IV 3 [27], 25.11-12, Plotin semble suggérer que le sujet de la mémoire est doté de passivité, cela ne signifie donc pas nécessairement qu'il soit sujet à altération et à changement. En réalité, comme on va le voir bientôt, Plotin vise à distinguer de l'activité autoréflexive de l'Intellect l'activité, imparfaitement unifiée, de ce qui se remémore. Il convient donc d'exposer, sans entrer dans les détails, la différence qui, selon Plotin, sépare le mode de pensée non discursif propre à l'Intellect du mode de pensée propre à l'âme qui s'est détachée de l'Intellect.

\section{L'âme et la mémoire}

La pensée de l'Intellect n'est pas dans le temps et n'implique pas la distinction de moments successifs; une telle succession ne prend place qu'au moment où l'âme (notoirement décrite en III 7 [45], 11.15 comme une "nature agitée ", $\left.\varphi \dot{\sigma} \sigma \iota \varsigma \pi 0 \lambda u \pi \rho \alpha ́ \gamma \mu \omega \nu^{10}\right)$ se sépare de son origine. À la différence de l'Intellect, le mode d'être de l'âme, imparfaitement unifié, implique la succession temporelle (III 7 [45], 11.35-40). Mais la pensée non discursive de l'Intellect comporte d'autres aspects distinctifs que l'atemporalité, aspects qui ont fait l'objet d'une discussion approfondie de la part de Eyjólfur K. Emilsson dans un livre récent ${ }^{17}$. En simplifiant beaucoup, voici quels sont les points principaux : le mode de pensée non discursif de l'Intellect (1) est non inférentiel ; (2) est «tout entier d'un seul coup »; (3) il pense ses objets tous ensemble ; (4) il est véridique et certain ; (5) il ne cherche pas son objet, mais le possède. Il n'y a pas dans l'Intellect de véhicules de la connaissance qui soient distincts des objets connus par leur moyen : en lui, les véhicules des pensées et les choses qui sont objet de pensée doivent être compris comme une seule et unique chose. Partant, la vérité d'un tel type de connaissance ne résulte pas de sa conformité à un objet extérieur: les pensées de l'Intellect, en effet, sont vraies dans la mesure où à travers elles est connu quelque chose qui n'est autre que ces pensées elles-mêmes. Par conséquent, l'Intellect n'a pas besoin de démonstrations ni de confirmations qui lui soient extérieures, mais il est manifeste pour lui-même; sa vérité n'est pas un accord avec quelque chose d'extérieur, mais avec elle-même (V 5 [32], 2.15-20).

C'est précisément ce mode de connaissance, dans lequel il n'y a aucune succession et dont les contenus sont parfaitement immanents à 
l'Intellect qui les pense, qui - affirme Plotin en IV 3 [27] - exclut la mémoire. En fait, la distinction entre Intellect et âme ne sépare pas une réalité "active» et une réalité "passive», mais deux formes différentes d'activité, dont une seule, celle de l'Intellect, est parfaitement autoréflexive. L'âme, qui est le sujet de la mémoire, n'est pas susceptible d'affections physiques, mais son activité implique malgré tout (1) qu'elle ait des contenus organisés selon une succession; (2) que ces contenus ne soient pas parfaitement immanents à ce qui les pense, mais soient tirés d'autre chose (d'une façon qui, quelle qu'elle soit, ne doit absolument pas être conçue sur un mode corporel ou physicalisée). C'est, me semble-t-il, à ce dernier aspect que fait allusion la formule é $\pi \iota x \tau \dot{\tau} \tau$ ou $\tau \iota v o ́ \varsigma$ de IV 3 [27], 25.11 : la mémoire est toujours mémoire de «quelque chose d'acquis »; sa présence exclut la parfaite immanence du contenu sur lequel s'exerce la mémoire. Pour ce qui est de la spécification de ce contenu, Plotin se borne pour le moment à affirmer que ce peut être soit une connaissance apprise ( $\mu \alpha \vartheta \dot{\eta} \mu \alpha \tau o \varsigma)$, soit une affection $(\pi \alpha \vartheta \dot{\eta} \mu \alpha \tau o \varsigma)$; la mémoire peut donc avoir un contenu intellectuel ou sensible.

Aussi élémentaire et schématique qu'elle soit, cette caractérisation est loin d'être dépourvue d'intérêt. Il est important de remarquer que Plotin ne montre aucune hésitation à affirmer que la mémoire se rapporte aussi à des connaissances ( $c f$. aussi IV 3 [27], 29.6-7, etc.). Il ne fait donc nullement sienne la prudence manifestée par Aristote quant à la possibilité que la mémoire se rapporte à des objets de connaissance et non pas seulement à des sensations. Comme on le sait, pour Aristote, les objets de la pensée ne sont objets de la mémoire que par accident (Mem. 1, 450a2225 ; 2, 451a28-29) ; la mémoire appartient par soi à la «faculté sensible première », c'est-à-dire à cette partie de l'âme à laquelle appartient aussi l'imagination. On ne peut s'attarder ici sur la doctrine aristotélicienne et sur ses difficultés bien connues, qui à plusieurs reprises ont retenu l'attention des interprètes ${ }^{18}$. Il suffira d'observer la différence, au moins dans le ton, entre la thèse formulée de façon synthétique en IV 3 [27], 25 et la position du De memoria.

Rien d'étonnant à cela : Plotin, en tant qu'exégète de Platon, ne pouvait pas ne pas tenir compte de la thèse célèbre formulée dans le Ménon, selon laquelle « chercher et apprendre ne sont au total que réminiscence » (81d ${ }^{19}$. Plotin a sans aucun doute parfaitement présente à l'esprit la doctrine platonicienne de la réminiscence ; d'autre part, la réminiscence ne

18. La bibliographie est importante; je me borne à signaler deux études récentes: Castagnoli 2006 ; Sassi 2007 (b). Il existe une récente édition commentée du De memoria et reminiscentia : Bloch 2007. Voir aussi le commentaire classique de Sorabji 2006.

19. Cette thèse a fait l'objet d'innombrables discussions. Pour un examen récent de la question, of. Scott 1999. 
peut pas être considérée sans autre précision comme une forme de mémoire $^{20}$. Plotin en est bien conscient et, de fait, en IV 3 [27], 25 il distingue la mémoire ( $\mu \nu \eta \dot{n} \mu \eta$ ), liée au temps et se rapportant à des contenus acquis «de l'extérieur », de la réminiscence ( $\alpha \dot{\alpha} \alpha \mu \nu \eta \sigma \iota \varsigma)$, entendue comme la réactualisation au sein même de l'âme de connaissances innées qu'elle possède par nature : «Il s'agit, dans ce cas, d'une espèce différente de mémoire; et pour cette raison le temps n'appartient pas à la mémoire ainsi définie. » (25.33-34.) On devrait pouvoir conclure de là que la mémoire de la connaissance acquise, dont Plotin parle en IV 3 [27], 25.11, est d'une autre nature que la réminiscence. En réalité, de telles distinctions sont fort difficiles à définir : quelle peut être exactement la différence entre la mémoire d'une connaissance apprise (25.11) et l'anamnèse (25.33-34), c'est quelque chose qui n'est pas clair.

En premier lieu, il ne semble pas y avoir place, dans la gnoséologie plotinienne, pour une connaissance entièrement acquise ; Plotin, en effet, ne fait jamais sienne une gnoséologie de type empiriste ou abstactionniste. Au contraire, dans le traité V 3 [49] il affirme que l'âme reçoit de l'Intellect «toutes choses, comme des caractères inscrits en elle » (V 3 [49], 4.22) ; les Formes reçues dans l'âme sont utilisées comme règle du jugement. Quand Plotin fait référence à une connaissance " acquise », en IV 3 [27], 25.11, c'est probablement au type de connaissance propre à la raison discursive qui, à la différence de celle qui appartient à l'Intellect, implique la succession et n'est pas auto-réflexive. La question de savoir si la connaissance discursive, ainsi conçue, coïncide purement et simplement avec l'anamnèse est difficile à trancher; c'est une question difficile à résoudre, que l'on peut ici laisser de côté (en effet, l'anamnèse semble impliquer non seulement que les Formes dans l'âme soient activées, mais que l'activité de l'âme soit orientée vers elles) ${ }^{21}$. Contentons-nous de relever que, quoi qu'il en soit, la connaissance discursive implique toujours la présence et l'actualisation de Formes innées qui sont utilisées comme critères du jugement sur les réalités empiriques; la connaissance discursive n'est donc pas séparable de la réminiscence ${ }^{22}$. D'ailleurs, la distinction entre mémoire et réminiscence, affirmée en IV 3 [27], 25.27 sqq., est en réalité, dans la suite du développement, plutôt laissée dans

20. Voir en dernier lieu Gonzalez 2007, notamment p. 284 n. 12 ; Centrone 2008, p. 116-117.

21. Cf. infra n. 34.

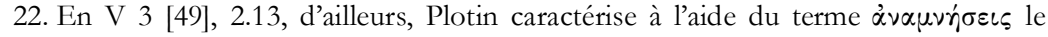
processus cognitif typique de l'âme discursive: les images reçues de la sensation sont adaptées aux «traces » des Formes présentes dans l'âme. Sur ce type discursif d'anamnèse et sa distinction par rapport à l'anamnèse dont il est question en IV, 3 [27], cf. Chiaradonna (à paraitre c). 
l'ombre. Comme l'a observé à juste titre Chiara Guidelli, «non seulement Plotin parle souvent de la mémoire de nature intellectuelle dans les termes de l'“anamnèse" platonicienne mais, par delà les distinctions ter-

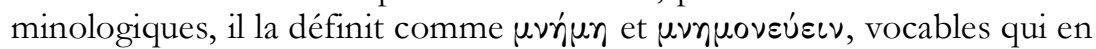
viennent ainsi à désigner le souvenir en général, incluant aussi bien la connaissance intellectuelle que la connaissance sensible $»^{22 a}(c f$. IV 4 [28], 4.14-20).

En fait, une des principales difficultés de ce chapitre est que, là plus qu'ailleurs, Plotin emploie des distinctions et des classifications sans trop se préoccuper de les définir et de les délimiter de façon précise. Il propose plutôt, pour les soumettre à examen, diverses thèses, sans se soucier de rigueur, parfois comme de simples hypothèses qui sont développées ou abandonnées sans donner trop d'importance à leur complète explicitation (pensons, en particulier, aux distinctions introduites en IV 3 [27], 29-31 à propos de la faculté imaginative, $\varphi \alpha \nu \tau \alpha \sigma i \alpha)$. Tout cela confère à l'exposition un caractère particulièrement aporétique et cryptique. Il y a probablement une raison précise à cette difficulté, et cette raison réside dans l'objet particulier qu'examine Plotin : il s'agit, il est bon de le répéter, non pas de la mémoire, mais de l'âme en tant que sujet de la mémoire. Or c'est précisément au moment où Plotin s'attache à examiner la relation entre l'âme et la mémoire que se dévoile pleinement le caractère ontologiquement «fluide» de l'âme puisque, sous la diversité de ses aspects et de ses facultés, elle traverse toute la hiérarchie ontologique, de l'Intellect jusqu'au monde physique. Dans la section qui nous occupe ici, Plotin parle explicitement de plusieurs niveaux de l'âme et, en IV 3 [27], 27.1-3, il distingue en nous deux «âmes»: celle qui est dite «la plus divine », en fonction de laquelle nous sommes proprement nous-mêmes,

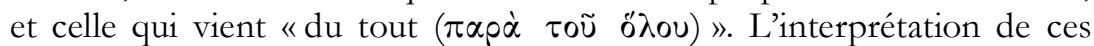
lignes est controversée ${ }^{23}$, mais il paraît raisonnable de conclure que pour Plotin il y a «en nous » (c'est-à-dire dans le mode d'être de notre âme ${ }^{24}$ )

22a. Guidelli 1988, p. 76-77.

23. Pour un excellent commentaire, voir Remes 2007, p. 23-32.

24. Plotin emploie souvent le pronom «nous » dans un sens presque technique, qui trouve sa première origine dans le Premier Alcibiade de Platon (128e), mais dont l'importance dans la pensée de Plotin ne peut être expliquée seulement comme un emprunt au texte de Platon. En simplifiant un peu, on peut dire que le pronom «nous» indique en général (mais pas toujours) l'âme individuelle discursive. En désignant le mode d'être de l'âme discursive à l'aide du pronom «nous », Plotin veut évidemment suggérer que c'est là la condition dans laquelle se trouve en général l'âme des hommes, ce qui, cependant, ne signifie pas du tout que tel soit pour eux l'unique mode d'être possible, ni le meilleur. Marzolo 2006, p. 161 et 164-175 (ad I 1 [53], 10.1-5 et 11.4), met en évidence, à juste titre, que «nous » est parfois employé par Plotin, pour se référer non à notre condition cognitive ordinaire, tournée vers le sensible («l'homme dans son expérience commune »), 
une double nature : il y a d'un côté l'âme pure, rattachée au monde intelligible, et de l'autre l'âme incarnée qui, tout en demeurant une nature incorporelle, n'en est pas moins liée à l'univers sensible et à l'âme qui le gouverne. Non seulement la nature de l'âme est composée de niveaux multiples, mais sa structure n'est en outre pas donnée une fois pour toutes : douée d'un dynamisme intérieur, elle est capable de se redéfinir et de se transformer, en fonction de l'aspect ou de la faculté qui en devient le centre unificateur. Comme nous le verrons, ce point aussi est pleinement mis en lumière quand Plotin discute du rapport entre âme, pensée discursive et connaissance des intelligibles. Une précision supplémentaire s'impose à ce sujet. Dans l'analyse du rapport entre âme et mémoire, s'entrecroisent au moins deux distinctions. La première est la distinction entre la perception et les autres facultés de l'âme auxquelles de toute façon est associé le souvenir. À cet égard, Plotin affirme clairement que la mémoire ne porte pas seulement sur les perceptions, mais aussi sur des mouvements intérieurs de l'âme qui n'impliquent pas la perception d'un objet «extérieur» (par exemple le désir de ce dont on n'a pas pu jouir : IV 3 [27], 26.35 sqq.) et sur des connaissances rationnelles. Il suit de là que la mémoire est déjà une activité d'ordre intellectuel et qu'elle doit donc être rigoureusement distinguée de la simple conservation d'impressions sensibles. Cette première thèse anti-empiriste ne suffit cependant pas à rendre compte du rapport complexe entre âme et mémoire, parce qu'elle se double d'une seconde distinction, laquelle ne sépare pas la perception des autres facultés propres à l'âme discursive, mais divise le complexe des facultés de l'âme auxquelles est associé le souvenir (c'est-à-dire l'âme discursive dans son intégralité, qui est le sujet aussi bien de la mémoire des perceptions que de la mémoire intellectuelle et de la réminiscence) de ce qui au contraire, en vertu de l'activité autoréflexive et atemporelle qui lui est propre, ne peut pas se remémorer. Il s'agit, comme on l'a vu plus haut, du Dieu intelligible, mais aussi (et c'est là le caractère distinctif de la conception plotinienne) de la partie supérieure de l'âme qui, selon une thèse célèbre de Plotin, ne quitte jamais l'Intellect et en partage le type de vie et le mode de connaissance ( $c f$. IV 8 $[6], 8)^{25}$. Le fait que, dans l'acte de se souvenir, l'âme discursive exerce une capacité active et ne soit pas sujette à des affections sensibles (à tel point que la mémoire peut porter non seulement sur des perceptions

mais au noyau supérieur et intelligible de l'âme : voir surtout, en ce sens, VI 4 [22], 14.1621. On trouvera plus de détails dans Chiaradonna 2008 (b). Une intéressante analyse philosophique du «nous» plotinien, dont je ne partage cependant pas entièrement les conclusions, se trouve dans Aubry 2004 ; voir aussi Aubry 2008.

25. La doctrine plotinienne de l'âme non descendue s'est trouvée au centre d'un vaste débat parmi les spécialistes; pour une vue d'ensemble, $c$. Chiaradonna 2005. 
mais aussi sur des désirs et des connaissances) n'exclut pas qu'elle soit pourtant, dans son ensemble, toujours distincte de cette "partie» de l'âme qui ne se remémore pas parce qu'elle exerce la forme la plus parfaite et la plus complète d'activité, qui exclut quelque forme de mémoire que ce soit (y compris la réminiscence).

En V 3 [49], Plotin, en quelques lignes célèbres, décrit ce qu'est « notre » condition, c'est-à-dire la condition dans laquelle se trouve ordinairement notre âme. Le centre de son activité est constitué par la faculté rationnelle, tournée vers le sensible, lequel fait l'objet de jugements qui reposent sur le critère a priori des «empreintes » laissées dans l'âme par les Formes intelligibles. Ce que «nous» sommes se situe entre les deux pôles que sont l'Intellect et la perception :

Les activités de l'Intellect ont leur origine en haut, celles de la sensation en bas, et voilà ce que nous sommes : la partie principale de l'âme, au milieu de deux puissances, l'une inférieure, l'autre meilleure ; inférieure est celle de la sensation, meilleure celle de l'Intellect. (V 3 [49], 3.36-4026.)

La mémoire correspond parfaitement à cette condition médiane. En IV 3 [27], 29-31, Plotin associe en effet la mémoire à une faculté bien précise, la $\varphi \alpha \nu \tau \alpha \sigma i \alpha$ (" faculté imaginative »), à laquelle il attribue principalement deux fonctions. Dans le cas de la mémoire liée à la sensation, la faculté imaginative conserve l'image de ce qui est perçu, même en son absence : «Si donc l'image de ce qui est absent est déjà dans cette faculté,

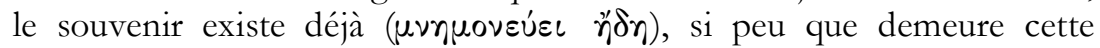

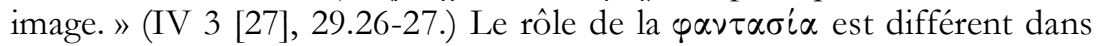
le cas des pensées. Comme on le sait, dans le De anima et dans le De memoria, Aristote avait affirmé qu'il n'est pas possible de penser sans images. Dans le contexte de la gnoséologie aristotélicienne, la signification d'une telle position est clairement abstractionniste: les objets de pensée sont en effet contenus «dans les formes sensibles » (De an. III, 8, 432a5), qui à leur tour sont contenues dans les sensations et préservées dans les images ( $\varphi \alpha \nu \tau \dot{\alpha} \sigma \mu \alpha \tau \alpha$, De an. III, 8, 432a9). La faculté intellective pense les formes « dans les images» (De an. III, 7, 431b2); celle-ci, autrement dit, n'est capable de saisir les von $\alpha \dot{\alpha}$ que là où ils sont présents dans les images des sens, actuelles ou imprimées dans la mémoire ${ }^{27}$. Plotin semble se référer à une conception de ce genre au début de IV 3 [27], 30 (1. 1-5), mais il passe ensuite rapidement à l'exposition d'une thèse différente sur le rapport entre pensée et $\varphi \alpha \nu \tau \alpha \sigma i \alpha$ : une thèse qui est en

26. Ce passage célèbre a été commenté à de nombreuses reprises par les interprètes : cf. en particulier Hadot 1980 et, plus récemment, Morel 2002.

27. Cf. Castagnoli 2006. 
fait diamétralement opposée à la thèse aristotélicienne et qui s'identifie avec sa vision particulière de la réminiscence ${ }^{28}$. Comme on l'affirme en 30.14-15, en effet, nous " pensons toujours, mais nous ne le comprenons

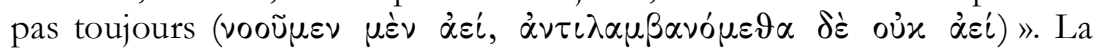
thèse qui est à l'arrière-plan de ces lignes consiste à attribuer à l'imagination la fonction de rendre consciente, en la faisant parvenir à toute l'âme, une activité qui autrement n'en intéresserait que la partie supérieure. Il n'y a évidemment aucune place pour un empirisme ou un abstractionnisme dans une telle conception, qui peut au contraire être caractérisée comme radicalement innéiste. Les pensées sont depuis toujours «en nous ", même si nous n'en sommes pas conscients; le fait de parvenir à la $\varphi \alpha \nu \tau \alpha \sigma i \alpha$ n'indique rien d'autre que l'appréhension explicite de quelque chose qui nous appartient par nature.

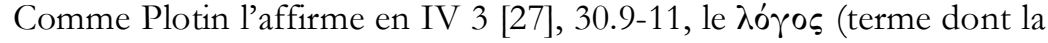
traduction est en général controversée, et qui semble indiquer ici une sorte de première articulation de la pensée noétique ${ }^{29}$ dévoile le contenu

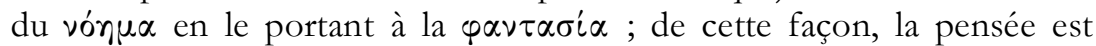
montrée "comme dans un miroir» (30.10) et devient objet d'appréhension consciente $\left(\dot{\alpha} \nu \tau \dot{\imath} \lambda \eta \dot{\psi} \psi \varsigma \varsigma_{s}\right)$ ou de souvenir $(\mu \nu \eta \dot{\mu} \mu \eta)^{30}$. Au fondement de cette position se trouve une thèse formulée aussi ailleurs par Plotin : à l'âme de chacun appartient une activité de pensée dont nous ne sommes pas toujours conscients, et qui est appréhendée quand elle est communiquée à l'âme tout entière. Le rôle conceptuel de la faculté imaginative ne consiste donc pas à associer aux contenus noétiques une image dérivée de la sensation, mais à réveiller l'âme et à lui faire appréhender dans toutes ses parties cette activité noétique qu'elle exerce déjà (ou, pour mieux dire, qu'elle exerce toujours), même sans en être consciente :

En effet, le contenu de l'acte intellectif parvient jusqu'à nous lorsque, en descendant, il arrive à la sensation; en effet, nous ne connaissons pas tout ce qui se passe dans une partie quelconque de l'âme avant que cela parvienne à l'âme tout entière. (IV 8 [6], 8.6-931.)

En IV 3 [27], 31, Plotin en vient à parler de deux facultés imaginatives : elles appartiennent respectivement aux deux âmes distinguées en

28. Sur le rapport entre la conception d'Aristote et celle de Plotin, $c f$. Linguiti 20042005.

29. Cf. Peroli 2003, p. 275-276 n. 84 ; D’Ancona 2007, p. 87 n. 36.

30. L'analogie du miroir renvoie à Tim. $71 \mathrm{~b}$, où Platon caractérise le foie comme dense, brillant et par conséquent apte à refléter les pensées qui proviennent de l'intel-

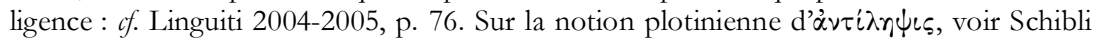
1989.

31. Sur le traité IV 8 [6], voir D’Ancona 2003. 
IV 3 [27], 27 et se rapportent, l'une aux sensibles, l'autre aux intelligibles ; à ces deux facultés imaginatives correspondent deux types différents de mémoire ${ }^{32}$. En outre, Plotin décrit une condition dans laquelle les «deux âmes » se trouvent en parfait accord l'une avec l'autre ; leurs facultés

32. Cf. IV 3 [27], 27.1-6; 31.1-5. Sur cette question, je renvoie à Linguiti 2004-2005 et à Gritti 2005, où l'on trouvera d'abondantes références textuelles et bibliographiques. Plutôt qu'à deux facultés distinctes, Plotin semble en réalité faire allusion à deux façons de procéder propres à la même faculté qui, lorsqu'elle est tournée «vers le bas », accueille en elle-même les contenus de la perception en leur donnant une configuration quasi intellective ( $c f$. IV 3 [27], 23.31-33), tandis que, lorsqu'elle est tournée "vers le haut », elle accueille les contenus noétiques, faisant en sorte qu'ils parviennent à la conscience de l'âme tout entière. J'incline à croire que, lorsque Plotin fait mention des souvenirs de l'âme supérieure, la plus divine, il ne fait pas allusion à la présence de la mémoire jusque dans l'âme située au niveau de l'intelligible, perpétuellement en contemplation des Formes. En effet, comme il l'explique en IV 4 [28], 4.15, on peut tout au plus dire que les souvenirs sont dans l'âme supérieure de manière potentielle, mais là-haut l'activité des Intelligibles obscurcit la mémoire ; les souvenirs passent donc à l'acte quand l'âme quitte (jamais intégralement) la région intelligible. Quand Plotin fait allusion, en IV 3 [27], 27.3 ; 31.1-2, aux souvenirs de l'âme supérieure, il peut se référer à la présence potentielle des souvenirs des Formes, obscurcie par l'activité de l'Intellect. Il est cependant tout aussi possible (et je tends à préférer cette explication) que les souvenirs de l'âme supérieure désignent l'activation du souvenir des Formes intelligibles qui a lieu au moment où l'âme supérieure se communique au reste des facultés psychiques en parvenant jusqu'à la faculté imaginative. En IV 3 [27], 30 et en I 4 [46], 10, Plotin soutient que, vis-à-vis des intelligibles, l'imagination joue le rôle d'un miroir; de cette façon elle rend consciente, en la faisant parvenir jusqu'à l'âme tout entière, une activité qui autrement n'en intéresserait que la partie supérieure, non descendue. En elle-même, cependant, la pensée de l'âme supérieure et non descendue est dépourvue d'images et de conscience : cf. IV 3 [27], 30.7-8 et 13-14 ; I 4 [46], 10.20-33 (Linguiti insiste avec raison sur le caractère anti-aristotélicien d'une telle position). Il parait cependant étrange que Plotin qualifie de propres (idias, 27.3) à l'âme supérieure, la plus divine, des souvenirs qui en réalité en proviennent sans pour autant en caractériser l'activité de manière intrinsèque. Il faut pourtant noter que, selon Plotin, quand les von $\tau \dot{\alpha}$ sont communiqués en eux-mêmes à une faculté inférieure, c'est moins cette dernière qui leur impose ses caractéristiques propres que les intelligibles qui régissent la faculté inférieure en lui permettant d'exprimer, pour autant que cela lui est possible, des contenus qui, structurellement, excèdent son mode de fonctionnement. C'est pour cette raison que Plotin peut affirmer, en IV 3 [27], 25.34-35, que le temps n'appartient pas à la réminiscence - c'est-à-dire à l'activation, dans l'âme descendue, des « remémorations » des Formes intelligibles qu'elle a déjà en elle en vertu de sa partie supérieure. Il

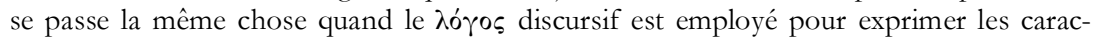
téristiques d'une nature (l'un-multiple intelligible) qui excède sa structure. Dans ce cas aussi, ce n'est pas le $\lambda o ́ \gamma o \zeta$ qui impose sa structure à la connaissance des intelligibles, mais c'est l'appréhension adéquate préalable des intelligibles qui régit l'emploi du $\lambda o ́$ yos, lui permettant d'exprimer, autant que possible, une connaissance extra-discursive. Cf. VI 5 [23], 5 et, pour plus de détails, Chiaradonna (à paraittre a). Ainsi s'explique, me semble-t-il, que Plotin puisse indiquer comme "propres » à l'âme supérieure les souvenirs qui en proviennent quand elle parvient jusqu'à la faculté imaginative, en régit le mode de fonctionnement et devient ainsi le centre unificateur de l'activité psychique tout entière. 
imaginatives ne sont pas séparées, et celle qui l'emporte est celle de l'âme la plus forte. Bien que de nombreux détails restent obscurs, il semble que cette condition soit propre à ceux qui ont activé en eux-mêmes le souvenir des intelligibles à tel point que leur âme est gouvernée par la partie supérieure qui en constitue, pour ainsi dire, le centre unificateur. Comme on l'a déjà dit, la structure de l'âme, qui comporte, dans leurs relations mutuelles, de nombreux niveaux d'activité, n'est pas donnée une fois pour toutes. En accord avec la doctrine platonicienne, selon laquelle l'âme est un principe dynamique et capable de mouvement spontané (cf. Pbaedr. 245c-246; Leg. X, 894b sqq.), Plotin pense qu'elle est capable de se redéfinir elle-même en déplaçant le centre de son activité d'une faculté à l'autre. Au moment où la connaissance intellectuelle est communiquée à l'âme tout entière, cette dernière est, pour ainsi dire, gouvernée par sa part supérieure ; l'exercice même de la sensation (inévitable aussi longtemps que l'âme est associée à un corps : cf. V 1 [10], 12.18-19) est unifié et régi en fonction de l'âme supérieure et intelligible, comme si «une faible lumière se diffusait à la faveur d'une plus forte » (IV 3 [27], 31.1213) ${ }^{33}$. Plotin clarifie ailleurs les conditions sous lesquelles peut avoir lieu ce processus de conversion au terme duquel, détournant notre attention des sensibles, nous devenons libres et capables d'appréhender les activités supérieures qu'exerce notre âme même quand nous n'en avons pas conscience (V 1 [10], 12) $)^{34}$.

\section{Mémoire et connaissance des intelligibles}

C'était une thèse bien établie dans le platonisme scolaire antérieur à Plotin, que la connaissance la plus haute que puisse atteindre l'âme incarnée s'identifie à l'activation des remémorations internes des Formes; on trouve exposée une doctrine semblable dans le Didaskalikos d'Alcinoos, généralement daté du $\mathrm{II}^{\mathrm{e}}$ siècle apr. J.-C35. L'origine de ces remémorations doit être cherchée dans la condition qu'a connue l'âme avant sa descente dans un corps, quand elle était soustraite aux conditionnements sensibles et capable de contempler directement les intelligibles transcendants. Bien que l'interprétation de la doctrine platonicienne de la réminiscence soit extrêmement controversée, on peut dire que cette conception trouve un fondement suffisant dans les textes, et en particulier dans le Phédon. Les deux thèses suivantes y sont formulées par Socrate: (1) l'âme, aussi longtemps qu'elle est associée à un corps, n'a pas d'accès direct à la connaissance des Formes; ce n'est qu'après la mort que nous

33. Voir, dans le même sens, I 4 [46], 10.10-21.

34. Sur ce chapitre discuté, $c f$. Atkinson 1983, p. 241-250.

35. Cf. Alcin. Didask. 155, 10 sqq. H. 
pourrons contempler «avec l'âme en soi les choses en soi » (Phaed. 66d), en pleine possession de ce dont, dans notre condition présente, nous

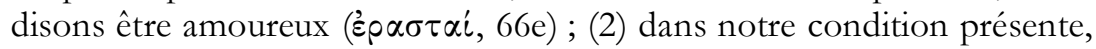
les jugements sur les réalités empiriques (par exemple les objets égaux) présupposent la présence en nous d'un critère a priori (l'Égal en soi) qui ne peut être tiré de ce qui est perçu; d'où il suit nécessairement qu'aient été connues auparavant $(\pi \rho \circ \varepsilon \iota \delta o ́ \tau \alpha, 74 \mathrm{e})$ les réalités auxquelles les objets perçus tendent à ressembler, sans jamais y parvenir entièrement. Cette connaissance a été acquise par l'âme avant sa venue dans un corps et, après avoir été perdue, au moins en partie, au moment de la naissance, elle peut être recouvrée indirectement dans le processus cognitif de la vie présente, assimilé à un ressouvenir (76c-77a).

Par rapport à l'exposition platonicienne de la réminiscence et par rapport aux exégèses qui en avaient été données antérieurement, il y a chez Plotin une différence, légère en apparence, mais lourde de conséquences. Aussi bien Platon que son exégète Alcinoos caractérisent la réminiscence en opposant un «maintenant » à un «avant »: selon le Socrate de Platon, au moment où s'active la mémoire innée des Formes, sont remémorées les choses que l'on a connues "dans un temps passé 》 ( $\alpha^{\prime} \pi \circ \tau \varepsilon$ है $\mu \alpha \vartheta \circ \nu$, Phaed. 76c); de manière analogue, Alcinoos distingue nettement l'intellection de «maintenant» ( $\tau \dot{\eta} \nu$ vũv, Didask. 155, 29 H.) de celle qui a lieu quand l'âme est séparée du corps. Quelle que soit la façon dont doivent être comprises de telles formulations, elles opposent sans équivoque deux états cognitifs hétérogènes : celui de l'âme incarnée, qui se souvient, et celui de l'âme avant sa descente dans le corps, qui contemple directement les Formes. Si l'on considère l'exposition plotinienne, une différence évidente apparaît aussitôt : à l'opposition entre «maintenant » et «avant» se substitue l'opposition entre ce que l'âme fait «toujours» (contempler les Formes intelligibles) et l'appréhension de cette activité,

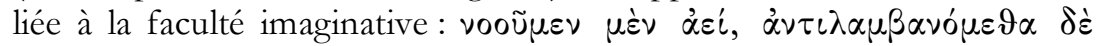
oủx $\alpha \dot{\alpha} i$. Tel est précisément le trait caractéristique de l'analyse de IV 3 [27] et 4 [28] : le fait d'être ou non dans un corps n'est pas présenté par Plotin comme une donnée de nature à conditionner l'accès de l'âme à la vision directe des Formes.

Plotin distingue, grosso modo, trois conditions où peut se trouver l'âme individuelle: l'âme incarnée dans un corps vivant; l'ombre de l'âme après la mort, qui conserve des souvenirs des événements qui ont eu lieu pendant sa vie dans un corps (l'ombre d'Héraclès dans l'Hadès dont parle Homère, $O d$. XI, 601 sqq., à laquelle fait allusion Plotin en IV 3 [27], 27) ; l'âme qui ne descend pas de l'intelligible et qui est parfaitement libre et seule (comparée par Plotin à l'Héraclès qui siège heureux parmi 
les dieux, dont il est question dans le texte homérique ${ }^{36}$. Encore une fois, l'analyse de Plotin est très compliquée et souvent obscure dans le détail. La thèse générale est en tout cas suffisamment claire : l'âme supérieure est toujours active; elle ne perd jamais contact avec l'intelligible, et il n'est pas nécessaire d'arriver au terme de notre vie corporelle pour nous joindre à elle et nous réapproprier notre activité cognitive supérieure. Plotin admet, naturellement, qu'une fois sortie du corps l'âme sera affranchie de beaucoup de choses «d'ici-bas » qui occupent sa vie sensible (IV 3 [27], 32.22-23). En fait, l'âme individuelle, aussi longtemps qu'elle est associée à un corps et assujettie au temps, ne peut jamais se libérer entièrement des conditionnements qui l'empêchent de s'établir de façon stable dans l'Intellect: le philosophe qui contemple l'intelligible aura pourtant toujours un corps, qui lui impose des besoins et des exigences impossibles à supprimer entièrement (cf. V 1 [10], 12.18-19). Pour cette raison Plotin observe ailleurs que «nous » pouvons perdre l'union avec le Nous; notre âme est toujours sujette à s'extérioriser et à redescendre vers la discursivité : «nous » ne sommes pas toujours tournés vers l'Intellect, alors que l'Intellect est toujours tourné vers lui-même ( $c f$. V 3 [49], 4.26$29 ; 9.22-23)^{37}$. Tout cela étant admis, il faut pourtant noter que Plotin ne nourrit aucun doute sur le fait que, aussi longtemps que l'âme est tournée vers les intelligibles et les contemple, cette connaissance est directe et adéquate, même ici-bas, sans attendre la fin de notre vie corporelle. Bref, la condition où se trouve la partie supérieure de l'âme ne change pas du fait que l'âme inférieure est descendue dans un corps, en "s'oubliant", en se séparant de l'Intellect qui est son origine ( $c f$. V 1 [10], 1.2) $)^{38}$. Que nous en soyons conscients ou non, il y a toujours dans notre âme "quelque chose » (cf. IV 8 [6], 6.3) qui reste dans l'intelligible et, même pour l'âme incarnée, il est possible de se joindre à sa partie supérieure en reprenant possession de son mode de vie et de sa connaissance les plus élevés : «... même ici-bas, quand elle désire réellement être là-haut, l'âme, tout en restant ici-bas, abandonne tout ce qui est étranger à ce monde d'enhaut.» (IV 3 [27]. 32.21-22.) Quelques lignes plus loin, Plotin se demande si l'âme, une fois terminé le cycle des incarnations et retournée définitivement dans l'intelligible, gardera la mémoire du fait qu'elle philosophait et que même alors, "quand elle était ici-bas, elle contemplait les choses d'en haut» (IV 4 [28]. 1.5-6).

Plotin, en somme, ne suggère pas du tout que la contemplation possible «ici-bas» ne soit qu'indirecte et donc d'une autre nature que

36. Cf. Pépin 1971. Voir aussi les passages plotiniens réunis et commentés dans Sorabji 2004, p. 269-274 (12[e]).

37. Cf. Hadot 1980 , p. 258-259 (ad V 8 [31], 1-9).

38. Voir à ce sujet D. Taormina, Plotino. L'anima e i suoi due oblii, en préparation. 
celle qui a lieu «là-haut ». En outre, à la différence de ce qui a été parfois suggéré ${ }^{39}$, la thèse du Phédon, selon laquelle l'âme incarnée n'a pas accès à la contemplation directe des Formes, n'a pas beaucoup d'écho dans l'exégèse plotinienne de Platon, fondée au contraire sur le principe opposé (et très difficile à retrouver tel quel dans les dialogues platoniciens) : quelque chose dans l'âme est toujours en contemplation directe des Formes, et l'itinéraire de la connaissance atteint son sommet dans la réappropriation de cette condition qui nous appartient toujours de droit, en vertu de notre partie supérieure non descendue de l'intelligible, même si, en fait, notre âme descendue dans un corps en a perdu la conscience. Tout cela est affirmé très nettement dans la section que nous considérons ici : "concernant les intelligibles, il est possible, comme on l'a dit, même à qui reste ici-bas, d'en parler au moyen de la même faculté qui a la capacité de contempler les choses d'en haut. » (IV 4 [28]. 5.6-8.)

Admettre tout cela implique qu'il est effectivement possible, pour chacun de nous, dès cette vie, sur un mode purement intellectuel et en vertu des facultés propres de notre âme, de participer d'une condition identique à celle dans laquelle se trouve le Dieu intelligible; comme Plotin l'affirme sans hésitation en I 2 [19], 6.2-3: «l'effort ne consiste pas à être exempt de faute, mais à être un dieu ${ }^{40}$. » Les interprètes se sont arrêtés à diverses reprises sur les multiples implications éthiques et épistémologiques de la doctrine plotinienne de l'âme non descendue, à l'égard de laquelle même les néoplatoniciens postérieurs (Jamblique et Proclus en particulier) firent preuve de prudence et de réserve ${ }^{41}$. Ce qu'il importe de souligner pour la présente discussion, c'est que l'activité cognitive de la partie supérieure de l'âme n'est pas assimilable au souvenir. La mémoire ne commence que lorsque l'âme individuelle se détache (quoique jamais

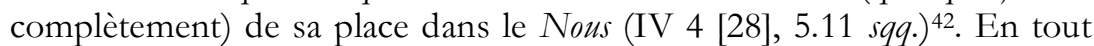
cas, la façon dont Plotin s'exprime suggère que la séparation du monde sensible n'est pas liée à la fin de notre vie corporelle ; au contraire, il est possible dès ici-bas de rentrer en possession de la condition cognitive supérieure qui rend en fait superflu tout type de souvenir, y compris la réminiscence des Formes. On a soutenu que «pour Plotin, comme pour Platon, nous connaissons les Formes grâce à une fonction cognitive qui

39. Cf. Gerson 1994, p. 279 n. 34. Selon Gerson, Plotin nierait que l'âme incarnée puisse avoir un accès aux Formes qui soit direct et indépendant de la perception : je ne crois pas que cette interprétation soit correcte. Voir les critiques fondées de Remes 2007, p. 147.

40. Cf. Dillon 1996. Sur le traité plotinien Sur les vertus, voir Catapano 2006.

41. Sur ce débat néoplatonicien, $c f$. Steel 2006.

42. Cf. D'Ancona 2007, p. 85. 
ressemble au souvenir ${ }^{43}$. Une telle conclusion ne peut cependant être acceptée qu'à la condition d'ajouter que, pour Plotin, la possibilité que nous entrions en contact avec les Formes ne se réduit nullement à la réminiscence ${ }^{44}$. Cette dernière est plutôt un préliminaire à l'acquisition d'un état cognitif supérieur qui comporte la contemplation directe des intelligibles. Du fait de la réminiscence, l'âme rentre en possession d'une condition parfaitement extérieure au temps et au devenir, condition qui lui appartient depuis toujours et dans laquelle nous connaissons les Formes exactement comme les connait le Dieu intelligible. Pour cette raison, Plotin peut affirmer sans hésitation que «la mémoire, même quand c'est celle des choses les meilleures, n'est pas la chose la meilleure » (IV 4 [28], 4.6-7) ${ }^{45}$.

43. Ibid. p. 73.

44. Comme on le sait, d'ailleurs, même pour Platon la connaissance des Formes ne passe pas toujours par l'intermédiaire de la réminiscence; par exemple, cette dernière semble ne jouer aucun rôle dans l'épistémologie des livres centraux de la République (il y a là-dessus un ample débat; je me limite à renvoyer à la discussion de Kahn 2006, en particulier p. 129-131, qui argumente en faveur d'une compatibilité substantielle entre l'épistémologie de la République et celle des dialogues où il est fait référence à la réminiscence). Sur la relation entre réminiscence et appréhension directe des Idées voir aussi Centrone 2008, p. 113. Je ne m'arrête pas sur la question, soulevée en particulier par Th. Ebert, relative à l'existence même d'une théorie platonicienne de la réminiscence qui, selon cet interprète, devrait être comprise en un sens purement métaphorique, comme un expédient rhétorique destiné à clarifier la nature du processus d'apprentissage ( $c f$. en dernier lieu Ebert 2004, p. 200-202). Pour un récent examen critique de cette lecture, $c f$. Gonzalez 2007, et, contre la lecture métaphorique de la section sur la réminiscence dans le Ménon, Scott 2006, p. 121-122.

45. Selon D'Ancona 2007, p. 84-85, l'argumentation de IV 4 [28], 1-2, qui tend à distinguer du souvenir la connaissance directe et atemporelle des intelligibles par l'âme, serait préparée par la distinction entre deux modalités de souvenir (l'une, associée à l'affection, propre à la partie inférieure de l'âme, et une autre qui survient $\alpha \dot{\pi} \alpha \vartheta \tilde{\omega} \varsigma$, propre à l'âme supérieure), formulée par Plotin à la fin de IV $3[27]:$ « [...] c'est cette observation qui prépare le développement argumentatif suivant [...] tout ce développement (scil. celui qui est relatif à l'intuition directe et atemporelle des intelligibles) est préparé par la distinction effectuée ici entre actes cognitifs moins parfaits, qui s'accompagnent de $\pi \dot{\alpha} \vartheta \circ \varsigma$, et actes cognitifs plus parfaits, qui en sont exempts. » Je ne partagerais pas cette conclusion. En fait, l'adverbe $\alpha \dot{\alpha} \alpha \vartheta \tilde{\omega} \varsigma$ revient en IV 3 [27], 32.3, mais sans indiquer (comme semble au contraire le suggérer D'Ancona) le souvenir de contenus purement cognitifs dépourvus de toute implication perceptive (à savoir, si je comprends bien, les Formes). Plotin envisage à cet endroit une question différente. En 31.16-18, il observe que l'âme supérieure, une fois sortie du corps, ne conserve que quelques souvenirs de l'âme inférieure, cependant qu'elle en abandonne d'autres. À titre de confirmation, il rappelle la

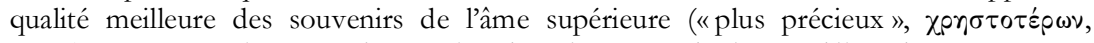
31.19) par rapport à ceux qui sont abandonnés et remplacés. Au début de IV 3 [27], 32 est mentionnée une objection possible : ce qui a été dit vaut-il aussi pour le souvenir de nos amis, de nos enfants, de notre épouse ou de notre patrie («tout ce dont il ne serait pas absurde que se souvienne un homme de valeur») ? À cette question, Plotin répond, 
Il est naturel de se demander quelle est la relation entre la réminiscence, décrite en IV 3 [27], 20-31 comme l'appréhension dans la faculté imaginative des contenus noétiques que l'âme pense toujours, et la connaissance parfaite des Formes que l'âme se réapproprie. Plotin ne s'arrête sur ce problème qu'au début de IV 4 [28], 5, en quelques lignes extrêmement obscures :

Quant aux intelligibles, est-ce que la même faculté par laquelle nous nous en souvenons les fait passer à l'acte ? En réalité, si nous ne les avons pas déjà contemplés, il s'agit de la mémoire ; autrement, il s'agit de la faculté par laquelle nous contemplions aussi là-haut. Cette faculté, en effet, est

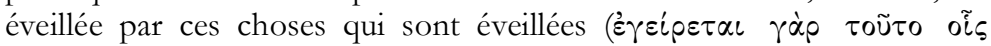

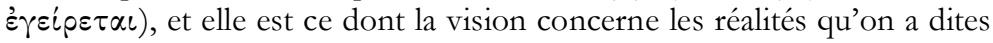
(scil. les intelligibles). (IV 4 [28], 5.1-5.)

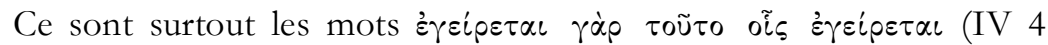
[28], 5.3-4) qui font difficulté. Il apparaît avec une clarté suffisante que la faculté qui est éveillée, c'est celle de contempler directement les intel-

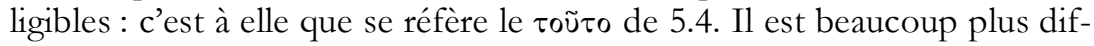
ficile de savoir à quoi se rapportent les mots oís è $\gamma \varepsilon i \rho \varepsilon \tau \alpha \iota$. J'inclinerais pour ma part à penser qu'il s'agit des intelligibles eux-mêmes ${ }^{46}$. Plotin, autrement dit, semblerait affirmer que l'activation des contenus noétiques présents dans notre âme (c'est-à-dire la réminiscence) comporte l'éveil de la faculté capable de les contempler directement et sur le mode approprié. Comme on le sait, le traité IV 8 [6] s'ouvre sur une description de l'état dans lequel, "s'éveillant de son corps à lui-même», le philosophe qui a écrit les Ennéades (parlant ici à la première personne)

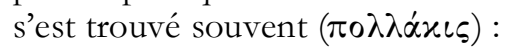

\footnotetext{
dans un premier temps, en opérant une distinction entre la façon dont ces souvenirs sont présents dans l'âme inférieure (c'est-à-dire «accompagnés d'affection", $\mu \varepsilon \tau \dot{\alpha} \pi \dot{\alpha} \vartheta_{0}$ ৩ $)$ et celle dont ils sont présents dans l'homme de valeur, à savoir $\alpha \dot{\pi} \alpha \vartheta \tilde{\omega} \varsigma$. Il est impossible d'interpréter cette précision comme si elle se rapportait à l'anamnèse des intelligibles. La distinction entre souvenirs liés à une affection et souvenirs dépourvus d'affection (32.3) est au contraire une observation préliminaire qui, dans les lignes suivantes et dans les premiers chapitres de IV [28], se voit préciser davantage et de façon définitive, en un sens qui tend à exclure la présence de tout souvenir (qu'il soit ou non lié à des affections) dans l'âme qui s'est complètement réunie avec sa partie la meilleure et la plus haute, dans la contemplation directe des Formes ( $f$. IV 4 [28], 1.11-14). Pour cette raison, on peut admettre, en suivant D'Ancona, que la distinction entre deux types de souvenirs " prépare » la suivante, mais seulement comme une étape préliminaire destinée à être dépassée, et non comme un élément sur lequel se fonderait la suite de l'argumentation et qu'elle présupposerait.

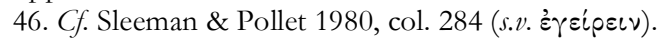


Bien des fois, m'éveillant de mon corps à moi-même et devenant extérieur aux autres choses, intérieur au contraire à moi-même, dans la vision d'une beauté si extraordinaire et ayant la certitude d'appartenir, surtout à ce moment, à la partie la meilleure de moi-même en me trouvant exercer le genre de vie le plus noble, ne faisant qu'un avec le divin et me trouvant fondé en lui, ayant procédé vers cet acte et m’étant placé moi-même audessus de tout autre intelligible... (IV 8 [6]. 1.1-747.)

Il semble raisonnable de conclure que la réminiscence coïncide avec le moment de l'éveil, où nous prenons conscience de notre partie supérieure dont l'activité est ainsi appréhendée par notre âme tout entière. Il est d'une importance cruciale de comprendre que, à la suite de cet éveil, ce n'est pas la faculté supérieure qui est limitée par les facultés inférieures auxquelles elle se rend accessible mais, au contraire, c'est l'âme tout entière qui est régie par sa partie supérieure ${ }^{48}$. En conséquence, la réminiscence, l'éveil de notre moi empirique à sa partie idéale, n'est que la première étape dans l'exercice d'un type de pensée identique à celui qui est attribué au Dieu intelligible en IV 3 [27], 25.13 sqq. Si tout cela est exact, il devient possible de distinguer trois moments différents dans l'itinéraire qui mène à la connaissance directe des Formes. (i) Dans sa condition ordinaire, la raison discursive utilise les «empreintes » des Formes comme critère de jugement sur les réalités empiriques vers lesquelles elle oriente son activité ; (ii) détournant son attention des sensibles, elle (scil. la raison discursive) la tourne vers les contenus noétiques qui sont accueillis dans la $\varphi \alpha \nu \tau \alpha \sigma i \alpha$; (iii) en conséquence de cela, la raison discursive se réunit à la faculté la plus haute de l'âme, à savoir la pensée non discursive, la contemplation directe des Formes. En (iii), l'âme de chacun s'élève complètement au-dessus de la discursivité ${ }^{49}$. Comme l'observe Paulina Remes, « ... une fois que l'on a comme il faut pris conscience de soi-même en tant qu'intellect et que l'on a obtenu une relation plus directe au royaume intelligible, il peut être possible, au moins pour de fugaces moments, de fonctionner sans que des représentations s'interposent entre qui pense et l'objet de ses pensées $»^{50}$. La pensée non discursive, non inférentielle (IV 4 [28], 5.6), de l'âme supérieure coïncide avec le type de pensée selon lequel «là-haut» aussi sont contemplés les êtres authentiques. Comme il arrive d'autres fois dans les argumentations

47. Je m'appuie sur la traduction italienne citée supra n. 31.

48. Cf. supra n. 32.

49. Quelque chose de semblable au passage de (ii) à (iii) est formulé par Plotin en V 3 [49], 8.37-57: cf. Chiaradonna 2008 (b).

50. Remes 2007, p. 123 : « ... once one has realised oneself correctly as intellect and gained a more direct relation to the intelligible realm, it may be possible, at least for passing moments, to function without representations that mediate between the thinker and the object of thoughts. » 
de Plotin, la fin d'une analyse (IV 4 [28], 5) ramène de cette façon à ce qui a été dit dans sa partie initiale (IV 3 [27], 25) : tout comme le Dieu intelligible, l'âme aussi a accès à une activité cognitive supérieure à n'importe quel type de mémoire.

Les difficultés d'une telle conception ne sont que trop évidentes: s'éveiller du corps à soi-même signifie, en toute rigueur, accéder à une condition extérieure au temps, mais il est vraiment difficile de comprendre quel sens peut avoir une telle condition pour notre moi empirique; encore plus difficile est-il de comprendre comment une expérience «transformative $»^{51}$ de ce type peut survenir «souvent» (et donc conformément à des catégories temporelles), comme Plotin l'affirme en IV 8 [6], 1.1, et comment il est possible que notre âme, après s'être réunie à la partie supérieure et intelligible d'elle-même, perde cette condition et revienne à s'orienter vers ses activités inférieures, liées à la discursivité et à la perception (chose inévitable, de fait, aussi longtemps que nous sommes dans un corps). Plotin lui-même, tout de suite après avoir décrit avec tant de force, dans les premières lignes de IV 8 [6], l'expérience de l'éveil de l'âme à elle-même, se déclare incapable d'expliquer comment, après avoir expérimenté une telle condition, il peut revenir à la pensée discursive et à la vie associée à un corps :

[...] une fois que, après ce répit dans le monde divin de l'intellect, je suis descendu à la raison discursive, je ne peux m'expliquer comment il peut se faire que je descende une fois encore et de quelle façon mon âme a jamais pu venir me trouver à l'intérieur du corps, si elle est cette même chose qui s'est révélée être en elle-même et par elle-même bien qu'elle se trouve dans un corps. (IV 8 [6], 1.8-11.)

Probablement faut-il renoncer à la prétention de tout expliquer : la doctrine de la contemplation directe des intelligibles constitue, avec celle de l'union extatique avec l'Un, une sorte de clé de voûte dans la structure de la philosophie de Plotin, dans laquelle l'argumentation philosophique s'unit de façon indissoluble à l'expérience directe. Certaines considérations d'Arthur H. Armstrong sont éclairantes à ce sujet:

Plotin [...], sur la base de sa propre expérience, savait parfaitement qu'il était deux personnes. L'expérience de s'éveiller à sa vraie nature et de se découvrir habiter de plein droit le monde de l'intelligence pure, une expérience vraiment toute différente de l'expérience quotidienne de son moi ici-bas, lié à un corps et plongé dans les soucis et désirs terrestres p. 167.

51. Sur l'aspect «transformatif» de la philosophie de Plotin, voir Burnyeat 2005 
[...] le conduisit, je crois, à cette conviction de la dualité de l'homme qui est exprimée de façon si imagée en VI 4 [22] 14, brièvement et clairement placée dans un contexte cosmique en II 3 [52] 9 et finalement affirmée avec force en I $1[53]^{52}$.

La thèse selon laquelle il est possible de parvenir, dès ici-bas, à une connaissance totalement appropriée du monde intelligible est peut-être, en fait, celle qui caractérise plus qu'aucune autre le platonisme de Plotin ${ }^{53}$. Elle est exposée dans les deux chapitres initiaux du traité VI 5 [23], où est énoncé ce qu'on peut définir comme le programme métaphysique de Plotin : concevoir les intelligibles à partir des principes qui leur sont adéquats (VI 2 [23], 2.5-6), sans faire usage en aucune façon des catégories adéquates à l'explication des $\operatorname{corps}^{54}$. La place de la mémoire dans la gnoséologie plotinienne doit être comprise dans le cadre de cette doctrine générale, selon laquelle il est possible, en principe, que l'âme s'affranchisse des conditionnements qui lui sont imposés par le temps et par la discursivité. Ces dernières précisions permettent, me semble-t-il, de réexaminer de façon synthétique le rapport entre la théorie de la mémoire de Plotin et la réflexion d'Augustin. Malgré tous les points de contact évidents qui ont été plusieurs fois relevés par les interprètes, il importe de remarquer que les thèses des deux philosophes sont très différentes. Le Dieu intelligible plotinien, en effet, est l'objet de la connaissance la plus haute, à laquelle notre âme peut accéder grâce à sa partie supérieure et non descendue (et donc sur un mode purement intellectuel, grâce au plein exercice de ses facultés). Plotin aurait eu beaucoup de difficultés à faire sien le célèbre exorde du livre $\mathrm{V}$ du De Trinitate, où est nettement affirmée l'insuffisance de notre pensée à saisir pleinement la nature de Dieu «à laquelle nous devons penser sans cesse, et à laquelle nous ne pouvons penser d'une façon qui en soit digne (de quo sempre cogitare debemus, et de quo digne cogitare non possumus) $\aleph^{55}$. Peut-être n'est-il

52. A.H. Armstrong 1974, p. 190 : «Plotinus [...], on the strength of his own experience, knew perfectly that he was two people. The experience of waking up to his true nature and finding bimself to be a rightful inhabitant of the world of pure intelligence, really a quite different kind of experience to his everyday experience of his self here below, body-bound and immersed in earthly concerns and desires [...] led, I believe, to that conviction of man's doubleness which is so picturesquely expressed in VI 4 [22] 14, shortly and clearly stated in a cosmic context in II 3 [52] 9 and strongly and finally asserted in I 1 [537. »

53. Comme je cherche à le montrer dans Chiaradonna 2009.

54. Pour plus de détails, voir Chiaradonna (à paraître b).

55. Il est vrai que Plotin situe au sommet de la réalité un principe qui excède notre connaissance, à savoir l'Un, supérieur à l'Être et à l'Intellect. Si l'Un, cependant, ne peut être connu de nous, ce n'est qu'en raison du fait qu'il est absolument simple et supérieur à toute connaissance : l'Un lui-même n'est pas à même de se connaitre lui-même ; il n'a ni pensée ni conscience de soi ( $c f . \mathrm{V} 6$ [24], 5.4-5; V 3 [49], 13.11-12; VI 9 [9], 6.48-50). 
nullement déraisonnable de suggérer que, à une conception différente de la connaissance de Dieu, correspond une différence radicale dans les conceptions de la mémoire élaborées par les deux philosophes ${ }^{56}$.

L'âme s'unit à l'Un en se vidant de tout contenu et de toute forme. Ainsi se tourne-t-elle vers l'Un et se fait-elle "posséder» par lui (VI 9 [9], 7.16 sqq.). La pensée (même la pensée auto-réflexive et parfaitement unifiée) n'entre plus dans cette expérience. L’Un n'est donc pas ce que nous ne pouvons connaître de façon adéquate en raison de notre faiblesse, mais ce qui n'est susceptible d'être connu de qui que ce soit (ni de notre âme discursive, ni de l'Intellect, ni de lui-même).

56. Plotin semble particulièrement éloigné de la conception de la mémoire comme « cogitandi modus» formulée dans Aug. Trin. 11, 8, 14 (voir à ce sujet Cillerai 2007). 


\section{BIBLIOGRAPHIE}

Alesse, F., F. Aronadio, M.C. Dalfino, L. Simeoni, E. Spinelli 2008: Anthropine sophia. Studi di filologia e storiografia filosofica in memoria di Gabriele Giannantoni, Naples, 2008 (Elenchos, 50).

Armstrong, A.H. 1974: "Tradition, Reason and Experience in the Thought of Plotinus", dans Atti del convegno internazionale sul tema : Plotino e il Neoplatonismo in Oriente e in Occidente (Roma, 5-9 ottobre 1970), Rome, 1974 (Problemi attuali di sc. e di cultura Quad., 198), p. 171-194.

AtKinson, M. 1983 : Plotinus, Ennead V. 1. On the Three Principal Hypostases, Oxford, 1983 (Oxford Classical and Philosophical Monographs).

Aubry, G. 2004 : Plotin, Traité 53 (I, 1), Introduction, traduction et notes, Paris, 2004 (Les Écrits de Plotin).

- 2008: «Un moi sans identité? Le Hèmeis plotinien », dans Aubry \& Ildefonse 2008, p. 107-125.

Aubry, G. \& IldEFOnSE, F. 2008 : Le moi et l'intériorité, Paris, 2008 (Textes et traditions, 17).

BEIERWALTES, W. 1981 : Plotin. Über Ewigkeit und Zeit ('Enneade' III 7), übersetzt, eingeleitet und kommentiert, 3. erg. Aufl., Frankfurt a.M., 1981 (Quellen der Philosophie. Texte und Probleme, 3).

BénatouïL, Th., Trabattoni, F., VAn Riel, G. (à paraître) : Platonisme et aristotélisme.

BLOCH, D. 2007 : Aristotle on Memory and Recollection, Text, Translation, Interpretation, and Reception in Western Scholasticism, Leyde, 2007 (Philosophia Antiqua, 110).

Blumenthal, H. 1971 (a) : Plotinus' Psychology: His Doctrines of the Embodied Soul, La Haye, 1971.

— 1971 (b) : Soul, World Soul, and Individual Soul in Plotinus, dans Schuhl \& Hadot 1971, p. 55-66.

Brisson, L. 2005 : Plotin, Traités 27-29, Paris, 2005 (GF, 1203).

— 2006: «La place de la mémoire dans la psychologie plotinienne », Études platoniciennes, 3 (2006), p. 13-27.

BurnyeAt, M. 2005 : «Platonism in the Bible: Numenius of Apamea on Exodus and Eternity ", dans R. Salles (éd.), Metaphysics, Soul, and Etbics in Ancient Thought. Themes from the Work of Richard Sorabji, Oxford, 2005, p. 143-169.

CASTAgnOli, L. 2006 : «Memoria aristotelica, memoria agostiniana », dans G.A. Lucchetta \& U. La Palombara (éd.), Mente, anima e corpo nel mondo antico. Immagini e funzioni, Pescara, 2006 (La Biblioteca di scheria, 1), p. 141-160.

Catapano, G. 2006 : Plotino, Sulle virtù (I 2 [19]), introduzione, testo greco, traduzione e commento, prefazione di J.M. Rist, Pise, 2006 (Greco, Arabo, Latino : Le vie del sapere, 2).

Centrone, B. 2008 : «L'anamnesi nel Fedone tra conoscere e sapere», dans Alesse, Aronadio, Dalfino, Simeoni, Spinelli 2008, p. 105-117.

Chiaradonna, R. 2002 : Sostanza movimento analogia. Plotino critico di Aristotele, Naples, 2002 (Elenchos, 37).

- 2005 : «La dottrina dell'anima non discesa in Plotino e la conoscenza degli intelligibili», dans E. Canone (éd.), Per una storia del concetto di mente, vol. 1, Florence, 2005 (Lessico Intellettuale Europeo, 99), p. 27-49.

- 2008 (a) : «Movimento dell'Intelletto e movimento dell'anima in Plotino (Enn. VI 2 [43], 8.10) ", dans Alesse, Aronadio, Dalfino, Simeoni, Spinelli 2008, p. 497-508.

- 2008 (b) : «Plotino: il "noi" e il Nous (Enn. V 3 [49], 8, 37-57)», dans Aubry \& Ildefonse 2008 , p. $277-293$. 
- 2009 : Plotino, Rome, 2009 (I Pensatori, 3).

— (à paraître a) : "L'analogia del cerchio e della sfera in Plotino", dans P. Totaro \& L. Valente, "Sphaera": Forma, immagine e metafora tra medioevo ed età moderna, Florence.

— (à paraitre b) : «Plotino e la scienza dell'essere », dans Bénatouil, Trabattoni, Van Riel.

— (à paraître c) : « La conoscenza dell'anima discorsiva », dans M. Barbanti (éd.), Anima e libertà in Plotino: Atti del Convegno, Catania.

Cillerai, B. 2007 : «Agostino : la memoria centro dell'actio animae », dans Sassi 2007 (a), p. $99-115$.

D’Ancona, C. 2003 : Plotino : La discesa dell'anima nei corpi (Enn. IV 8 [6]); Plotiniana arabica (Pseudo-Teologia di Aristotele, capitoli 1 e $7 ;$; Detti del sapiente greco 》, a cura di —, Padoue, 2003 (Subsidia Mediaevalia Patavina, 4).

- 2007 : «Plotino : memoria di eventi e anamnesi di intelligibili », dans Sassi 2007 (a), p. 67-98.

Dillon, J. 1996 : «An ethic for the late antique sage », dans L.P. Gerson (éd.), The Cambridge Companion to Plotinus, Cambridge, 1996 (Cambridge Companions to Philosophy), p. 315-335.

EberT, Th. 2004: Platon. Phaidon, Übersetzung und Kommentar, Göttingen, 2004 (Platon Werke, I 4).

Emilsson, E.K. 1988 : Plotinus on Sense-Perception. A Pbilosophical Study, Cambridge, 1988.

- 2007 : Plotinus on Intellect, Oxford, 2007.

Ferrari, F. 1995 : Dio idee e materia. La struttura del cosmo in Plutarco di Cheronea, Naples, 1995 (Strumenti per la ricerca plutarchea, 3).

FleEt, B. 1995 : Plotinus. Ennead III.6. On the Impassivity of the Bodiless, Translation and Commentary, Oxford, 1995.

Gerson, L.P. 1994 : Plotinus, Londres, 1994 (The Arguments of the Philosophers).

GonZALEZ, F.J. 2008: "How is the Truth of Beings in the Soul? Interpreting Plato's Anamnesis", Elenchos, 29 (2008), p. 275-301.

GRITTI, E. 2005 : «La phantasia plotiniana tra illuminazione intellettiva e impassibilità dell’ anima », dans R. Chiaradonna (éd.), Studi sull'anima in Plotino, Naples, 2005 (Elenchos, 42), p. 251-274.

Guidelli, C. 1988 : «Note sul tema della memoria nelle Enneadi di Plotino », Elenchos, 9 (1988), p. 75-94.

Hadot, P. 1980 : «Les niveaux de conscience dans les états mystiques selon Plotin », Journal de psychologie normale et pathologique, 77 (1980), p. 243-266.

Henry, P. \& H.-R. SChWyZER 1964 : Plotini Opera, I, Oxford, 1964 (OCT).

KAHN, Ch. 2006 : «Plato on Recollection», dans H.H. Benson (éd.), A Companion to Plato, Oxford, 2006 (Blackwell Companions to Philosophy), p. 119-132.

LinguiTi, A. 2004-2005 : «Immagine e concetto in Aristotele e Plotino », dans L. Cristante (éd.), Incontri triestini di filologia classica, 4 (2004-2005), p. 69-80 (Polymnia : studi di filologia classica, 6 ).

Majumdar, D. 2007 : Plotinus on the Appearance of Time and the World of Sense. A Pantomime, Aldershot, 2007.

Marzolo, C. 2006 : Plotino, Che cos'è l'essere vivente e che cos'è l'uomo ? I 1 [537, introduzione, testo greco, traduzione e commento, prefazione di C. D'Ancona, Pise, 2006 (Greco, Arabo, Latino : Le vie del sapere, 1).

Morel, P.-M. 2002 : "La sensation, messagère de l'âme. Plotin, V 3 [49], 3 ", dans M. Dixsaut (éd.), avec la collaboration de P.-M. Morel et K. Tordo-Rombaut, La Connaissance de soi. Études sur le Traité 49 de Plotin, Paris, 2002 (Tradition de la pensée classique), p. 209-227. 
- 2007 : Plotin, Traité 41, dans L. Brisson \& J.-F. Pradeau (éd.), Plotin, Traités 38-41, Paris, 2007 (GF, 1316).

PÉPIN, J. 1971 : «Héraclès et son reflet dans le Néoplatonisme », dans Schuhl \& Hadot 1971, p. 167-192.

Peroli, E. 2003 : Dio nomo e mondo. La tradirione etico-metafisica del Platonismo, Milan, 2003 (Temi metafisici e problemi del pensiero antico. Studi e testi, 91).

Pieretti, A. (à paraître) : Memoria e senso della vita. Dal mondo antico ad Agostino, Rome.

REMES, P. 2007 : Plotinus on Self. The Philosophy of the 'We', Cambridge, 2007.

SAssi, M.M. 2007 (a) : Tracce nella mente. Teorie della memoria da Platone ai Moderni. Atti del convegno, Pisa, Scuola normale superiore, 25-26 settembre 2006, a cura di —, Pise, 2007 (Seminari e convegni, 9).

- 2007 (b) : “Aristotele fenomenologo della memoria », dans Sassi 2007 (a), p. 25-46.

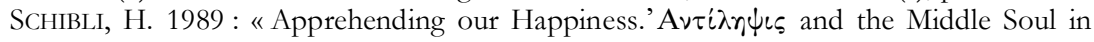
Plotinus, Ennead I $4.10 »$, Phronesis, 34 (1989), p. 205-219.

SCHUHL, P.-M. \& P. НADOT 1971 : Le Néoplatonisme: actes du colloque de Royaumont, 9-13 juin 1969, Paris, 1971 (Colloques internationaux du CNRS).

ScotT, D. 1999 : «Platonic Recollection», dans G. Fine (éd.), Plato, I: Metaphysics and Epistemology, Oxford, 1999 (Oxford Readings in Philosophy), p. 93-124.

- 2006 : Plato's Meno, Cambridge, 2006 (Cambridge Studies in the Dialogues of Plato).

Sleeman, J.H. \& G. Pollet 1980 : Lexicon Plotinianum, Leyde-Louvain, 1980 (Ancient and Medieval Philosophy. De Wulf-Mansion Centre, Series 1, II).

SorabjI, R. 2004 : The Pbilosophy of the Commentators (200-600 AD). A Sourcebook, vol. 1, Psychology (With Ethics and Religion), Londres, 2004.

- 2006: Aristotle on Memory, 2nd edition, Chicago, 2006.

STEEL, C. 2006 : Il Sé che cambia. L'anima nel tardo neoplatonismo: Giamblico, Damascio e Prisciano, édition italienne par L.I. Martone, Bari, 2006 (Biblioteca filosofica di Quaestio).

TAORMINA, D.P. (à paraître) : «La concezione plotiniana della memoria dei sensibili e la critica ad Aristotele », dans Bénatouil, Trabattoni, Van Riel (à paraittre).

Warren, E.W. 1965 : «Memory in Plotinus », The Classical Quarterly, 15 (1965), p. 252260. 